\title{
MDM2 309 T/ G Polymorphism Is Associated With Acute Myeloid Leukemia: A Meta- Analysis
}

\author{
Siqian Lu' ${ }^{1}$, Wenru Tang ${ }^{2}$, Ying Luo ${ }^{1 *}$ \\ ${ }^{1}$ Lab of Molecular Genetics of Aging \& Tumor, Faculty of Life Science and Technology \& Faculty of Environmental Science and Engineering, Kunming \\ University of Science \& Technology, 727 Jing Ming Nan Road, Chenggong County \\ ${ }^{2}$ Lab of Molecular Genetics of Aging \& Tumor, Faculty of Life Science and Technology \& Faculty of Environmental Science and Engineering, Kunming \\ University of Science \& Technology, 727 Jing Ming Nan Road, Chenggong County
}

Received: 24 December, 2016; Accepted: 16 January, 2017 ; Published: 26 January, 2017

*Corresponding author: Dr. Ying Luo, Lab of Molecular Genetics of Aging \& Tumor, Faculty of Life Science and Technology \& Faculty of Environmental Science and Engineering, Kunming University of Science \& Technology, 727 Jing Ming Nan Road, Chenggong County, Email: yingluo@kmust.edu.cn

\begin{abstract}
Objective:The $309 \mathrm{~T} / \mathrm{G}$ polymorphism of murine double minute 2 (MDM2) gene is associated with acute myeloid leukemia, but conflicting results have been reported. In this study, we performed a meta-analysis to estimate the association between MDM2 $309 \mathrm{~T} / \mathrm{G}$ polymorphism and acute myeloid leukemia risk.
\end{abstract}

Methods: Electronic search of PubMed was conducted to select case-control studies that contain available genotype frequencies of the MDM2 309 T/G polymorphism. Pooled Odds Ratio (OR) with 95\% Confidence Interval (CI) was used to assess the strength of the association.

Results: Six case-control studies, which consist of 1121 cases and 3974 controls, were identified. After pooling all the eligible studies in the meta-analysis, we found that the MDM2 $309 \mathrm{~T} / \mathrm{G}$ polymorphism was significantly associated with risk of acute myeloid leukemia (Recessive model G/G versus T-carriers: OR=1.738, 95\% CI: 1.424 $2.122, p=0.000$; additive model $\mathrm{G}$ versus T: OR $=1.298,95 \% \mathrm{CI}: 1.159$ $1.453, p=0.000$ ). Symmetrical funnel plot derived from Egger's test $(P=0.970)$ and Begg's test $(P=0.851)$ indicated the lack of publication bias.

Discussion: This meta-analysis suggests that individuals with T/G or G/G genotype of MDM2 SNP309 were significantly associated with increased acute myeloid leukemia risk. To draw comprehensive and true conclusions, well-designed studies with large sample sizes and representing different ethnicities are required.

Keywords: MDM2 T309G; meta-analysis; acute myeloid leukemia

\section{Introduction}

Acute Myeloid Leukemia (AML) is a cancer that starts in the bone morrow and is characterized by rapid growth of abnormal blood cells, which ultimately replace healthy hematopoietic cells and blood elements [1]. Patients with AML show higher risks of infections and bleeding than healthy individuals [2]. The majority of patients with AML possess genetic alterations, which disturb the normal mechanisms of the growth and maturation of blood cells [3]. Thus far, well-designed studies on genes related to AML remain insufficient.

Susceptibility genes in AML have been studied. MDM2, a disease-susceptibility gene, is over expressed in many human cancers [4]. MDM2, as a ubiquity in E3 ligase, can negatively reduce the stability of p53 [5]. A single nucleotide polymorphism (T-G exchange at nucleotide 309 in the first intron) located in the core promoter of the $M D M 2$ gene can increase the affinity of the transcription factor $S P 1$ to the $M D M 2$ core promoter; this phenomenon up regulates MDM2 expression, resulting in $\backslash$ attenuated p53 stress responses and enhanced tumor transformation and resistance to apoptosis [6]. Previous studies suggested that over expression of MDM2 is present in half of the total number of patients with AML [7]. Therefore, we hypothesize that the $M D M 2$ gene can be potentially used as a clinical biomarker in AML.

Several studies were conducted to investigate the potential association between the MDM2 $309 \mathrm{~T} / \mathrm{G}$ polymorphism and AML risk in humans $[2,8,9]$. However, published data show conflicting results. Therefore, we conducted this meta-analysis to quantitatively assess the effect of the MDM2 $309 \mathrm{~T} / \mathrm{G}$ polymorphism on the risk of AML.

\section{Materials and Methods}

\section{Publication search}

PubMed was searched using the terms 'MDM2 309', 'polymorphism' and 'acute myeloid leukemia'. Case-control studies containing available genotype frequencies of MDM2 309 were chosen. Additional studies were identified by a manual search of the references of original studies.

\section{Statistic analysis}

For control group of each study, the observed genotype frequencies of the MDM2 $309 \mathrm{~T} / \mathrm{G}$ polymorphism were assessed for Hardy-Weinberg equilibrium using the $\chi 2$ test. The strength 
of association between $309 \mathrm{~T} / \mathrm{G}$ polymorphism of $M D M 2$ gene and acute myeloid leukemia was accessed by calculating crude odds ratios (ORs) with 95\% Confidence Intervals (CIs). The pooled ORs were performed for dominant model $(G / G+G / T$ vs. TT), and recessive model (G/G vs. $\mathrm{G} / \mathrm{T}+\mathrm{T} / \mathrm{T})$, additive genetic model ( $G$ vs. T) respectively. Heterogeneity assumption was checked by a chi-square based Q-test. A P-value of $<0.05$ for the Q-test indicated a lack of heterogeneity among the studies, the summary OR estimate of each study was calculated by the random effects model [10]. The potential for publication bias was examined by a Begg's test and Egger's linear regression test $(\mathrm{P}<0.05$ considered representative of statistical significance) [11]. All statistical analyses were performed with Stata software (version9.0; Stata Corporation, College Station, TX).

\section{Result}

\section{Eligible studies}

We identified 6 case-control studies on the association between MDM2 309 T/G polymorphism and acute myeloid leukemia, which including 1121acute myeloid leukemia cases and 3974controls.These data were used in our meta-analysis (Table 1). The distribution of genotypes in the controls of all the studies was in agreement with Hardy-Weinberg equilibrium.

\section{Meta-analysis}

The results of the association between the MDM2 309 T/G polymorphism and acute myeloid leukemia and the heterogeneity test were shown in Table 2. As shown in Figure 1 and Figure 2, the recessive model (G/G vs. G/T+T/T) and additive model ( $G$ vs. $\mathrm{T})$ showed a significant association with acute myeloid leukemia risk (Recessive model G/G vs. T - carriers: $\mathrm{OR}=1.738,95 \%$ CI: $1.424 \sim 2.122, p=0.000$; additive model G vs. T: OR=1.298, 95\% CI: $1.159 \sim 1.453, p=0.000$, Table 2, Figure 1, Figure 2. As shown in Figure 3, there is no significant association was found in dominant model $(\mathrm{G} / \mathrm{G}+\mathrm{G} / \mathrm{T}$ vs. T/T: OR $=1.285,95 \% \mathrm{CI}: 0.988$ $\sim 1.669, p=0.061$, Table 2, Figure 3

\section{Publication bias}

Funnel plot and Egger's test were done to estimate the publication bias of literatures. The results of Egger's test provided statistical evidence for funnel plot symmetry (for G vs. T: $P=0.128$, GG vs. G/T+T/T: $P=0.970$ ) (Table 2 ), suggesting the absence of publication bias.

\section{Discussion}

The p53 pathway plays a significant role in the prevention of tumor formation, and MDM2 is an ubiquitin E3 ligase that negatively regulates the stability of p53 [5]. The proper regulation of MDM2 levels is vital for TP53 tumor suppression. Previous studies revealed thatSNP309 G/G cell lines expressed higher levels of MDM2 (eightfold mRNA and fourfold protein levels) than TT cell lines; moreover, intermediate protein levels (1.9-fold) were observed in heterozygous (T/G) cell lines [6]. Studies found that the MDM2 $309 \mathrm{~T} / \mathrm{G}$ polymorphism is associated with increased risk of many cancers [12]. Observed that patients with the MDM2 $309 \mathrm{~T} / \mathrm{G}$ polymorphism exhibit higher risk of esophageal
Table 1: The distribution of the MDM2 $309 \mathrm{~T} / \mathrm{G}$ polymorphism for cases and controls

\begin{tabular}{|l|l|l|l|l|l|l|l|l|l|}
\hline Author & year & \multicolumn{9}{l}{ Case } & \multicolumn{3}{l|}{ Control } & \multicolumn{2}{l|}{ Pa } \\
\cline { 3 - 11 } & TT & TG & GG & TT & TG & GG & \\
\hline $\begin{array}{l}\text { Nathan A. } \\
\text { Ellis(study in UK) }\end{array}$ & 2008 & 31 & 34 & 13 & 958 & 1027 & 286 & 0.676 \\
\hline $\begin{array}{l}\text { Nathan A. } \\
\text { Ellis(study in } \\
\text { USA) }\end{array}$ & 2008 & 35 & 40 & 14 & 330 & 303 & 88 & 0.156 \\
\hline Xiong, X & 2009 & 32 & 123 & 76 & 35 & 68 & 25 & 0.435 \\
\hline Phillips, C. L & 2010 & 176 & 178 & 78 & 194 & 229 & 62 & 0.661 \\
\hline Gamal T Ebid & 2012 & 21 & 33 & 14 & 30 & 29 & 6 & 0.789 \\
\hline $\begin{array}{l}\text { Anuradh } \\
\text { Cingeetham }\end{array}$ & 2015 & 50 & 76 & 97 & 73 & 150 & 81 & 0.828 \\
\hline
\end{tabular}

a:p value for Hardy-Weinberg equilibrium in control group.

Table 2: ORs and $95 \% \mathrm{CI}$ for acute myeloid leukemia risk and the MDM2 $309 \mathrm{~T} / \mathrm{G}$ polymorphism under different genetic models.

\begin{tabular}{|l|l|l|l|l|}
\hline $\begin{array}{l}\text { genetic } \\
\text { model }\end{array}$ & pooled OR [95\% CI] p & Heterogeneity & $\begin{array}{l}\text { Begg's } \\
\text { Test }\end{array}$ & $\begin{array}{l}\text { Egger's } \\
\text { Test }\end{array}$ \\
\hline $\begin{array}{l}\text { Additive } \\
\text { (G vs. T) }\end{array}$ & $1.298[1.159 \sim 1.453]<0.001$ & 0.111 & 0.573 & 0.128 \\
\hline $\begin{array}{l}\text { Recessive } \\
\text { (G/Gvs. }\end{array}$ & $1.738[1.424 \sim 2.122]<0.001$ & 0.577 & 0.851 & 0.97 \\
\hline $\begin{array}{l}\text { T-carriers) } \\
\text { Dominant } \\
\text { (G-carriers } \\
\text { vs. T/T) }\end{array}$ & $1.285[0.988 \sim 1.669] 0.061$ & 0.062 & 0.039 & 0.004 \\
\hline
\end{tabular}

squamous cell carcinoma compared with other genotype groups [13]. Found that the MDM2 $309 \mathrm{~T} / \mathrm{G}$ polymorphism is associated with increased risk of bladder cancer. Anuradh et al. [14] found that presence of MDM2 $309 \mathrm{G} / \mathrm{G}$ genotype at promoter region increased MDM2 gene expression, hence inhibiting the p53 stress response resulting in leukemic cell transformation. Further, their study indicated that the over expression of MDM2 may lead to cell vulnerability to chemotherapy due to p53 degradation. Therefore, the MDM2 309 T/G polymorphism could be associated with AML risk, with the $309 \mathrm{G}$ allele as the risk factor.

Previous studies on associations between MDM2 SNP309 and AML risk provided inconsistent results, and most of these studies involved less than a few hundred AML cases $[14,15,16,8,9]$, which is insufficient to reliably assess any genetic effects. As such, we performed this meta-analysis to provide up-to-date clinical evidence for adopting MDM2 SNP309 as a prognostic biomarker in patients with AML. Based on six case-control studies on MDM2 SNP309 and AML, the MDM2 $309 \mathrm{G}$ allele probably acts as an AML risk factor. We found that individuals with the MDM2 309G allele ( $G / G$ or $T / G)$ showed significantly higher risk of AML compared with those with the reference MDM2 309T/T genotype.

All the results in this study should be considered prudently 


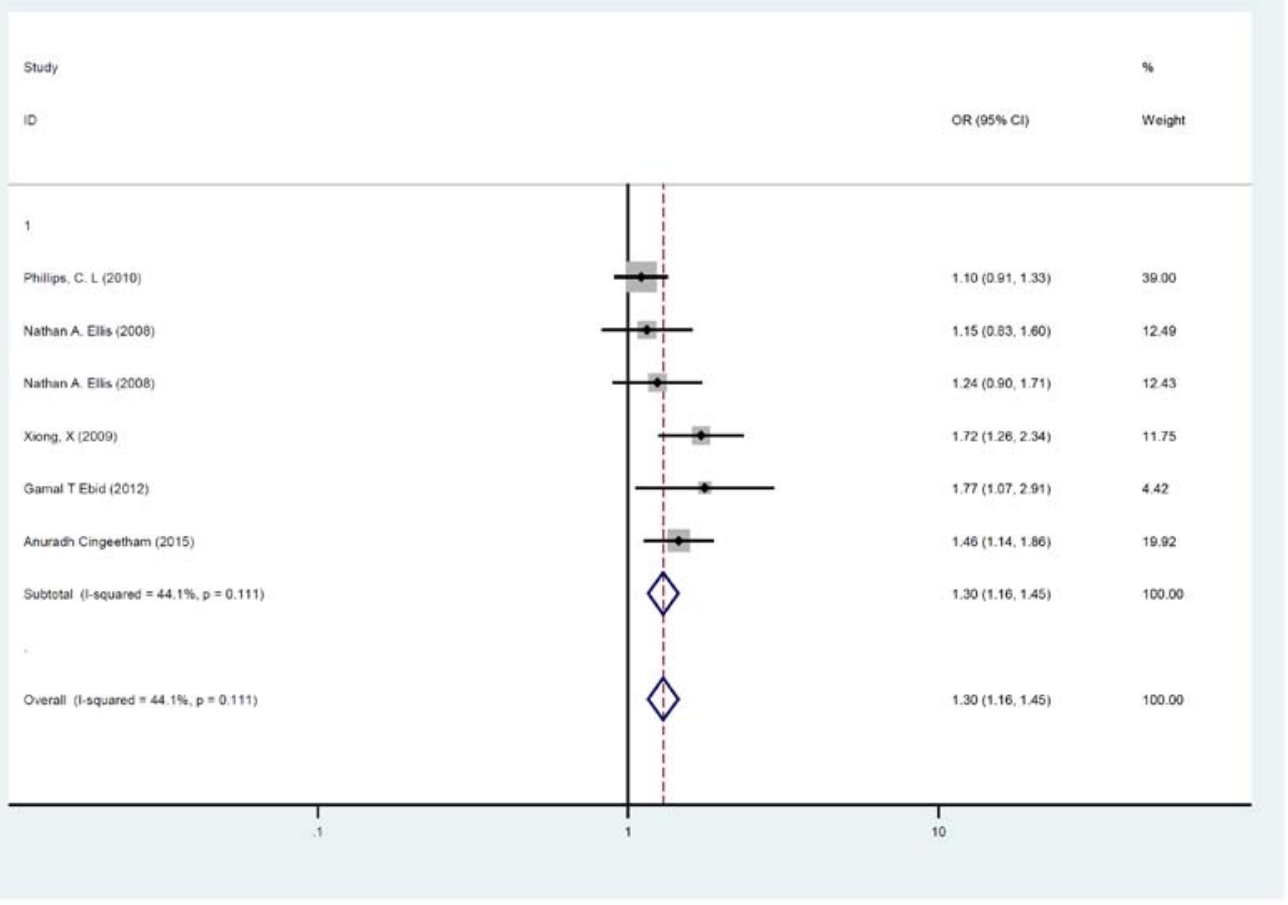

Figure 1: Forest plot of ORs of AMLG allele when compared to the T allele (Additive model) .The squares and horizontal lines correspond to the study-specific OR and 95\% CI. The area of the squares reflects the study-specific weight. The diamond represents the pooled OR and $95 \% \mathrm{CI}$.

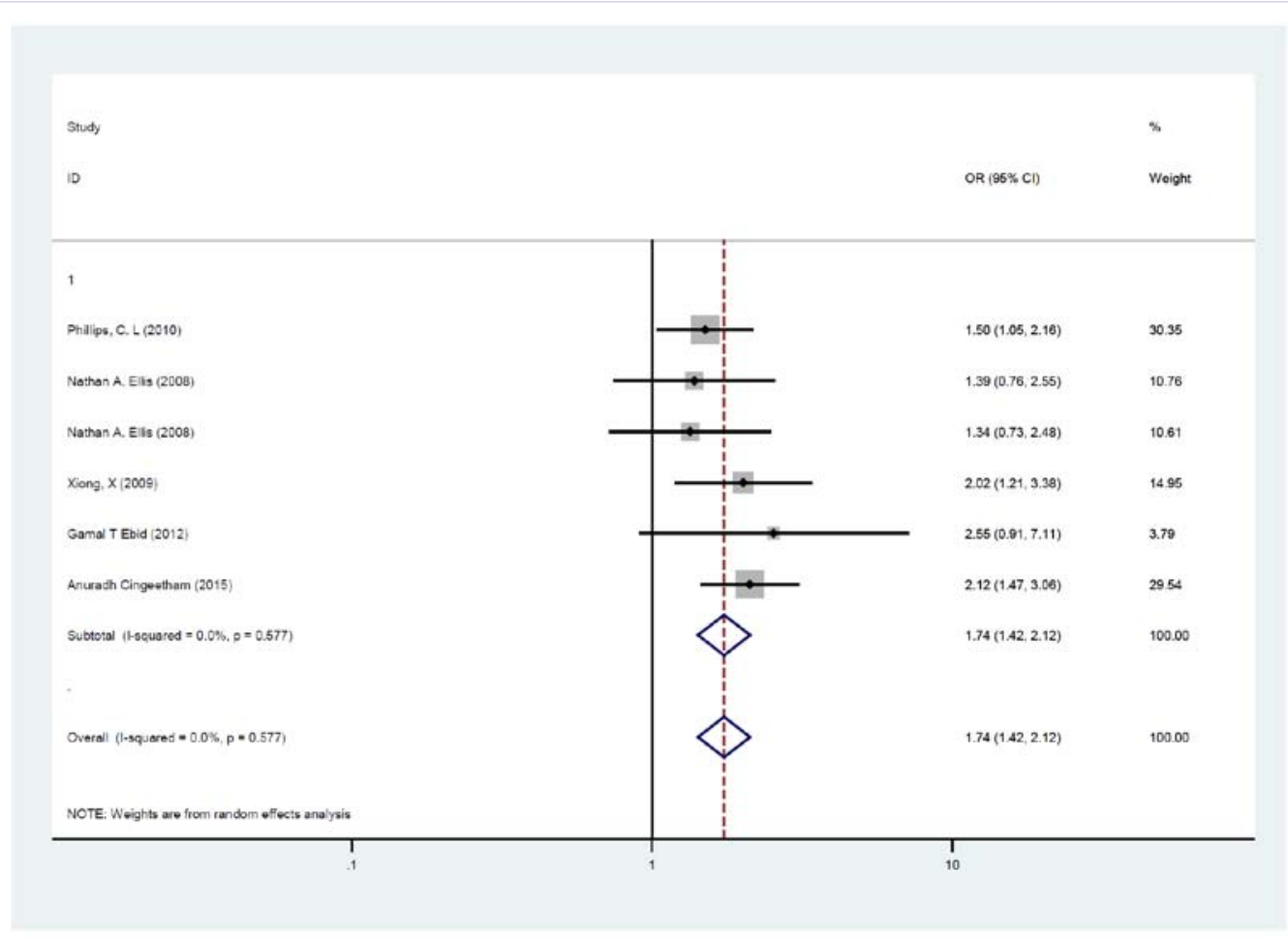

Figure 2: Forest plot of ORs of AMLG/G genotype when compared to the T allele carriers (G/T + T / T) (Recessive model). The squares and horizontal lines correspond to the study-specific OR and $95 \% \mathrm{CI}$. The area of the squares reflects the study-specific weight. The diamond represents the pooled $\mathrm{OR}$ and $95 \% \mathrm{CI}$ 


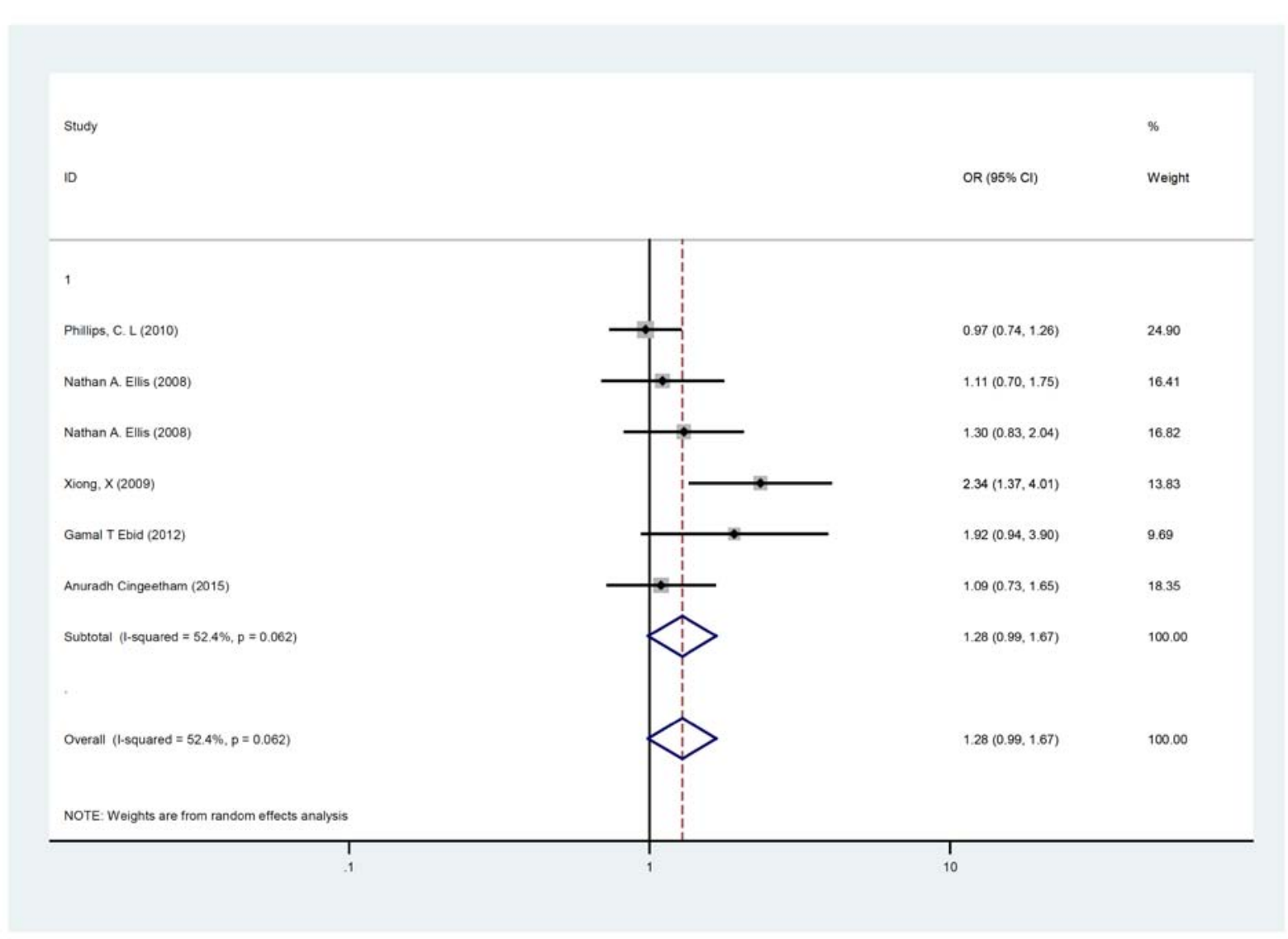

Figure 3: Forest plot of ORs of AMLG allele carriers $(G / G+G / T)$ when compared to the $T / T$ genotype (Dominant model). The squares and horizontal lines correspond to the study-specific OR and $95 \% \mathrm{CI}$. The area of the squares reflects the study-specific weight. The diamond represents the pooled OR and $95 \% \mathrm{CI}$

because of several limitations. The first limitation is the lack of adjustment according to detailed individual data, such as age, sex, and lifestyle. Second, the total sample size used in our metaanalysis is insufficient to draw a conclusion of the relationship between the MDM2 $309 \mathrm{~T} / \mathrm{G}$ polymorphism and AML risk. To achieve a more reliable conclusion, further analysis must be performed using adjusted individual data and a large sample size without significant publication bias.

In conclusion, this meta-analysis, which consists of six eligible studies (1121 cases and 3974 controls in all), indicates that MDM2309 G/G and T/G may act as an AML risk factor. Although some limitations exist, our metaanalysis can provide valuable information for studying the relationship between MDM2309 T/G polymorphism and AML.

\section{Acknowledgements}

This work was financially supported by the National Science Foundation of China (No. 30960152), the Nature Science Foundation of Yunnan Province (No. 2008C043M), and the Fund of State Key Laboratory of Genetics Resources and Evolution (No. GREKF10-07).

\section{Conflict of interest}

The authors declare no competing financial interests.References

1. Buccisano F, Maurillo L, Del Principe MI, Del Poeta G, Sconocchia G, Lo-Coco F, et al. Prognostic and therapeutic implications of minimal residual disease detection in acute myeloid leukemia. Blood. 2012;119(2):332-41. doi: 10.1182/blood-2011-08-363291.

2. Ellis NA, Huo D, Yildiz O, Worrillow LJ, Banerjee M, Le Beau MM, et al. MDM2 SNP309 and TP53 Arg72Pro interact to alter therapy-related acute myeloid leukemia susceptibility. Blood. 2008;112(3):741-9. doi: 10.1182/blood-2007-11-126508.

3. Radomska HS, Basseres DS, Zheng R, Zhang P, Dayaram T, Yamamoto Y,et al. Block of C/EBP alpha function by phosphorylation in acute myeloid leukemia with FLT3 activating mutations. J Exp Med. 2006;203(2):371-81.

4. Rayburn E, Zhang R, He J, Wang H. MDM2 and human malignancies: expression, clinical pathology, prognostic markers, and implications for chemotherapy. Curr Cancer Drug Targets. 2005;5(1):27-41.

5. Vogelstein B, Lane D, Levine AJ. Surfing the p53 network. Nature. 2000;408(6810):307-10. 
6. Bond GL, Hu W, Bond EE, Robins H, Lutzker SG, Arva NC, et al. A single nucleotide polymorphism in the MDM2 promoter attenuates the p53 tumor suppressor pathway and accelerates tumor formation in humans. Cell. 2004;119(5):591-602.

7. Seliger B, Papadileris S, Vogel D, Hess G, Brendel C, Storkel S, et al. Analysis of the p53 and MDM-2 gene in acute myeloid leukemia. Eur J Haematol. 1996;57(3):230-40.

8. Phillips CL, Gerbing R, Alonzo T, Perentesis JP, Harley IT, Meshinch $\mathrm{S}$, et al. MDM2 polymorphism increases susceptibility to childhood acute myeloid leukemia: a report from the Children's Oncology Group. Pediatr Blood Cancer. 2010;55(2):248-53. doi: 10.1002/pbc.22519.

9. Xiong X, Wang M, Wang L, Liu J, Zhao X, Tian Z, et al. Risk of MDM2 SNP309 alone or in combination with the $\mathrm{p} 53$ codon 72 polymorphism in acute myeloid leukemia. Leuk Res. 2009;33(11):1454-8. doi: 10.1016/j.leukres.2009.04.007.

10. Mantel N, Haenszel W. Statistical aspects of the analysis of data from retrospective studies of disease. J Natl Cancer Inst. 1959;22(4):71948.
11.Egger M, Davey Smith G, Schneider M, Minder C. BMJ. 1997;315(7109):629-34.

12. Hong Y, Miao X, Zhang X, Ding F, Luo A, Guo Y, et al. The role of P53 and MDM2 polymorphisms in the risk of esophageal squamous cell carcinoma. Cancer Res. 2005;65(20):9582-7.

13. Onat OE, Tez M, Ozcelik T, Toruner GA. MDM2 T309G polymorphism is associated with bladder cancer. Anticancer Res. 2006;26(5A):3473-5.

14. Cingeetham A, Vuree S, Jiwatani S, Kagita S, Dunna NR, Meka PB, Gorre,et al. Role of the MDM2 promoter polymorphism $(-309 \mathrm{~T}>\mathrm{G})$ in acute myeloid leukemia development. Asian Pac J Cancer Prev. 2015;16(7):2707-12.

15. Ebid GT, Sedhom IA, El-Gammal MM, Moneer MM. MDM2 T309G has a synergistic effect with P21 ser31arg single nucleotide polymorphisms on the risk of acute myeloid leukemia. Asian Pac J Cancer Prev. 2012;13(9):4315-20.

16. Falk IJ, Willander $\mathrm{K}$, Chaireti R, Lund J, Nahi H, Hermanson $\mathrm{M}$, et al TP53 mutations and MDM2(SNP309) identify subgroups of AML patients with impaired outcome. Eur J Haematol. 2015;94(4):355-62. doi: $10.1111 /$ ejh.12438. 